\title{
QUÍMICA VERDE: UN NUEVO RETO
}

\author{
GREEN CHEMISTRY: A NEW CHALLENGE \\ Nerlis Paola Pájaro Castro \\ Química Farmacéutica. Grupo de Química Ambiental y Computacional, Facultad de Ciencias Farmacéuticas. \\ Universidad de Cartagena. Campus de Zaragocilla. Cartagena, Bolívar, Colombia \\ npajaroc@unicartagena.edu.co \\ Jesús Tadeo Olivero Verbel \\ Químico Farmacéutico Ph.D. Profesor Titular. Grupo de Química Ambiental y Computacional, Facultad de Ciencias \\ Farmacéuticas. Universidad de Cartagena. Campus de Zaragocilla. Cartagena Bolívar, Colombia \\ joliverov@unicartagena.edu.co
}

Fecha de recepción: 23 de mayo de 2011

Fecha de aprobación: 19 de diciembre de 2011

\section{RESUMEN}

En la actualidad, existe un enorme deterioro del medio ambiente que ha generado la necesidad de buscar alternativas que conduzcan a la sostenibilidad ambiental. Una de estas herramientas es la "química verde", concepto que contempla el diseño de productos y procesos que reduzcan la generación de sustancias peligrosas y maximicen la eficiencia en la utilización de recursos materiales y energéticos. El empleo de tecnologías menos contaminantes, permitirá a las empresas químicas mitigar los efectos ambientales asociados a su actividad, reduciendo el consumo de materiales e incrementando la participación de recursos renovables. Para alcanzar estas metas, se han propuesto 12 principios básicos de química verde, aplicables en diferentes campos, tales como la medicina, la agricultura, la industria química y farmacéutica. Esta revisión detalla los principios y usos principales de la química verde, y su aplicación como una filosofía de trabajo para avanzar hacia un verdadero desarrollo sostenible.

Palabras clave: química verde, medio ambiente, contaminación, deterioro ambiental, sostenibilidad.

\begin{abstract}
Currently there is a significant environmental degradation that has generated the need to search for alternatives leading to environmental sustainability. One of these tools is "green chemistry", a concept that involves the design of products and processes that reduce the generation of hazardous substances and maximize the efficiency in the utilization of material and energetic resources. The use of less pollutant technologies will allow chemical companies to mitigate the environmental effects associated with their business, reducing material consumption and increasing the participation of renewable resources. To achieve these goals, 12 basic principles of green chemistry have been proposed, applicable to different fields, such as medicine, agriculture, and chemical and pharmaceutical industries. This review outlines the
\end{abstract}


principles and main uses of green chemistry, and their application as a philosophy of work to move towards a truly sustainable development.

Keywords: green chemistry, environment, pollution, environmental degradation, sustainability.

\section{INTRODUCCIÓN}

Problemas ambientales como el cambio climático, la contaminación del aire y de los recursos hídricos, la erosión, la deforestación, el declive de la biodiversidad, y el deterioro de la capa de ozono, entre otros, han avanzado de forma progresiva en los últimos años [1], afectando la calidad de vida de los habitantes del Planeta y la integridad de su patrimonio natural, lo cual ha generado la necesidad de promover acciones tendientes a preservar el medio ambiente. Gran parte de estos problemas se genera por procesos químicos, uso indiscriminado de recursos naturales, manejo inadecuado de residuos industriales, agrícolas y domésticos [2]. Por consiguiente, ha surgido un conjunto de acciones encaminado a reducir el deterioro ambiental, denominado "química verde" [3], [4], y [5]. Desde su inicio conceptual en 1991, la química verde ha crecido de forma continua nacional e internacionalmente, con la creación de organismos, redes, instituciones, revistas y programas educativos [4] y [6].

La implementación de la química verde tiende a reducir y eliminar sustancias peligrosas para el medio ambiente y la salud desde la industria química [5]. En este sentido, se han propuesto prácticas destinadas a favorecer la sostenibilidad del Planeta, mediante la conservación y uso racional de bienes y servicios medio ambientales, así como la planificación y administración eficiente de los recursos naturales por parte de las autoridades competentes, teniendo en cuenta tres aspectos fundamentales: el ambiente, la economía y la sociedad [4], [7] y [8], por ser estos, las bases de la sostenibilidad y aplicabilidad de los principios de la química verde.

En la actualidad, existen muchos estudios en los cuales se utiliza la química verde para reducir el impacto ambiental de los residuos industriales y lograr un manejo adecuado de los recursos ambientales [9]. Sin embargo, son muchos los retos pendientes a los cuales debemos dar respuesta, y por lo tanto es necesario seguir investigando sobre las interacciones ambientales en un rango de escalas espacial y temporal, teniendo en cuenta el ciclo de vida completo de los productos, desarrollar nuevas metodologías de detección, identificación y separación de contaminantes, mecanismos de los cambios fotoquímicos y los de toxicidad, impulsar las fuentes renovables de materias primas, adelantar estudios en el campo de la biomasa, y en la reutilización de residuos de las catálisis en las células solares fotovoltaicas [10].

\section{QUÍMICA VERDE}

La química verde fue adoptada como una propuesta novedosa para reducir y/o eliminar los problemas ambientales derivados de actividades industriales. Según la US Environmental 
Protection Agency (EPA), la química verde es el "uso de la química para la prevención de la contaminación, y el diseño de productos químicos y procesos benéficos para el ambiente" [3], [6], [7] y [8]. La química verde plantea 12 principios para conseguir sus objetivos [1], [5], [6], [11], [12], [13], [14] y [15]:

1. Prevenir la creación de residuos. Resulta más útil evitar o reducir la producción de desechos que tratarlos o limpiarlos tras su formación.

2. Maximizar la economía atómica. Los métodos sintéticos deben maximizar la incorporación de cada material utilizado en el proceso.

3. Realizar síntesis química menos peligrosa. Consiste en elaborar procesos que generen la mínima toxicidad e impacto ambiental.

4. Diseñar productos y compuestos menos peligrosos. Los productos químicos se deben diseñar con una toxicidad mínima.

5. Utilizar disolventes y condiciones seguras de reacción. Las sustancias auxiliares de los procesos químicos (disolventes, tampones, aditivos de separación, entre otros), han de ser inocuas y reducirlas al mínimo.

6. Diseñar para la eficiencia energética. Debe minimizarse los requerimientos energéticos para los procesos químicos, los cuales serán evaluados por su impacto medioambiental y económico, y reducirlos al máximo, intentando llevar a cabo los métodos de síntesis a temperatura y presión ambiente.

7. Utilizar materias primas renovables. Los materiales de partida utilizados deben proceder de fuentes renovables, en la medida en que sea económica y técnicamente factible.

8. Evitar derivados químicos. La síntesis debe diseñarse con el uso mínimo de grupos protectores para evitar pasos extras y reducir los desechos.

9. Utilizar catalizadores. Debe emplearse catalizadores lo más selectivos y reutilizables posibles.

10. Diseñar productos fácilmente degradables al final de su vida útil. Los productos químicos han de ser diseñados de tal manera que al culminar su función no persistan en el ambiente y puedan degradarse a derivados inertes o biodegradables.

11. Monitorear los procesos químicos en tiempo real para evitar la contaminación. Debe crearse sistemas de control y monitorización continuos para prevenir la producción de sustancias peligrosas durante los procesos.

12. Prevenir accidentes. Diseñar los procesos químicos, utilizando métodos y sustancias que reduzcan los accidentes (emisiones, explosiones, incendios, entre otros), y minimizar los daños cuando se produzca un accidente.

Los principios de la química verde fueron propuestos originalmente por Paul Anastas y John Warner en su libro Green Chemistry, theory and practice en 1998, y constituyen el pilar de la química verde. La aplicación de estas estrategias en la implementación de procesos innovadores, contribuirán a la sostenibilidad del Planeta en la sociedad, la economía y el ambiente [1 1 ].

Uno de los procesos que puede implementarse con base en la química verde, es la síntesis química por que puede optimizarla mediante condiciones adecuadas, reduciendo los 
requerimientos energéticos e incrementando la eficiencia de los procesos, con la catálisis y el diseño de sustancias químicas más seguras [6]. Por ejemplo: la oxidación de alcohol a grupo carbonilo, genera una cantidad significativa de residuos peligrosos. En consecuencia se ha desarrollado un método alternativo que evita el uso de reactivos tóxicos, aplicando los principios uno, tres y siete de la química verde [16]. Por otra parte, Trost y sus colaboradores demostraron cómo el uso de catalizadores maximiza la economía atómica (principio 2), usando una variedad de catalizadores de paladio en reacciones de alquilación alílica [16]. De forma similar, las macrolactonas pueden obtenerse a partir del correspondiente ácido carboxílico en un proceso catalizado por paladio, con un 100\% de eficiencia atómica y a temperatura ambiente, aplicando el principio 6 [16]. En forma análoga, los principio 4, 6 y 10, se aplican en la síntesis catalítica de PPT (polímero poliaspartato térmico), que se utiliza en la síntesis del ácido poliacrílico [16].

\section{APLICACIONES DE LA OUÍMICA VERDE}

El reto de la sostenibilidad del Planeta, está en encontrar nuevas tecnologías y procesos ambientales responsables, imprescindibles para la fabricación de productos que requiere la sociedad [6]. Los procesos químicos industriales necesitan compuestos y materiales que intervienen en la vida cotidiana y que repercuten en el entorno. Durante décadas, estos aspectos no se tuvieron en cuenta, pero en la actualidad todos los procesos deben ser diseñados con metodologías que prevengan la contaminación y sean seguras para los seres humanos y para el ambiente [3], [17] y [18]. Recientemente, se ha demostrado que la química verde puede utilizar materiales compatibles con el entorno, desarrollando procesos innovadores que reduzcan y/o eliminen la generación de sustancias peligrosas, residuos y tóxicos persistentes provenientes de diversas actividades industriales [1], [8], [13] y [18].

El uso de rutas alternativas para la síntesis de compuestos, es una de las herramientas que utiliza la industria química para reducir la emisión de sustancias contaminantes al ambiente, mejorando la calidad de vida de las personas que podrían verse afectadas [17]. Algunas incorporan procesos naturales como la síntesis fotoquímica y biomimética, el uso de materias primas inocuas y renovables, condiciones de reacción alternas, empleando solventes inofensivos para la salud y el ambiente, utilización de catalizadores que pueden ser fácilmente recuperados y reutilizados [19] y [20]. De este modo, se incrementa la selectividad de las reacciones, disminuyendo o suprimiendo los residuos y las emisiones contaminantes [4], [21] y [22]. La selección adecuada de las herramientas de síntesis es fundamental y determinante para la selectividad, reactividad química [11] e incremento de la eficacia [16], [20] y [23]. Actualmente, más del 80\% de las sustancias químicas son producidas, usando catálisis [24]. Sin embargo, los catalizadores y solventes empleados en ella, pueden ser tóxicos, carcinógenos y contaminantes ambientales.

Uno de los principios de la química verde propone el uso de solventes benignos, que no sean inflamables, tóxicos y no produzcan emisiones de compuestos orgánicos volátiles derivados de su uso como medio de reacción en la industria química y farmacéutica, con el fin de minimizar la 
producción de contaminantes y subproductos [25]. Por otra parte, la aplicación de tecnologías catalíticas que reduzcan la producción de residuos líquidos o sólidos, o su purificación, proporciona una fácil separación del producto, eliminando la necesidad de utilizar métodos de separación como la destilación o la extracción [26]. En concordancia con lo anterior, se han realizado estudios que proponen usar catalizadores y solventes en reacciones catalíticas bajo condiciones benéficas para el medio ambiente. Algunos ejemplos se presentan en la Tabla 1.

La industria utiliza solventes en grandes cantidades. Más del 50\% de los materiales usados en procesos farmacéuticos, corresponden a solventes orgánicos, algunos de los cuales son recuperados y reutilizados. Sin embargo, la mayoría son incinerados como método de disposición final [43], lo cual causa un gran impacto en términos económicos y ambientales. El uso de reacciones que utilicen menos solventes o prescindan de los mismos, es el más eficiente [12] y [27]. En forma análoga, el agua es un solvente que tiene muchas ventajas, incluida su ausencia de inflamabilidad y toxicidad, y es seguro para el ambiente [20] y [44].

Estudios recientes proponen otros solventes y catalizadores, tales como el dióxido de carbono supercrítico que se utiliza como catalizador y se considera como un buen "disolvente verde" en diferentes tipos de síntesis. Por ejemplo: en las reacciones de bromación de compuestos aromáticos y en las de polimerización del metacrilato de metilo. Entre las ventajas que presenta, se encuentran su alta difusibilidad, baja viscosidad y densidad intermedia, así como su baja toxicidad, inflamabilidad y empleo de baja temperatura [4], [12] y [18].

Los líquidos iónicos también son buenos catalizadores y disolventes que pueden reemplazar el diclorometano, por que no son volátiles, ni inflamables, eliminan los peligros asociados con solventes orgánicos volátiles, reducen el uso de energía y minimizan las emisiones [6], [12] y [45].

A pesar de todos estos avances, el desarrollo de métodos químicos verdes no sólo requiere de menos contaminación y residuos, sino también de cambios que proporcionen beneficios en términos del impacto ambiental global. El reto no es sólo usar sustancias verdes durante los procesos sino además, crear productos más amigables con el ambiente y que puedan sustituir los actuales, además de ser diseñados de tal forma que después de cumplir su función, no persistan en el ambiente y se degraden a compuestos inocuos [16].

El término química verde debe entenderse dentro del concepto fundamental de lo que es química y como tal, estudia la materia y todas sus transformaciones. Bajo este contexto, los químicos diseñadores de síntesis pueden elaborar sustancias químicas y transformaciones moleculares para modificar las propiedades fisicoquímicas indeseables de un compuesto peligroso (reacciones de adición, isomerización y transformaciones directas de la unión C-H) [3] y [20], mediante pequeños cambios en su estructura, de tal forma que puedan afectarse significativamente sus propiedades, como por ejemplo la biodegradabilidad, movilidad ambiental y toxicidad. El diseño verde busca generar productos muy efectivos y que además, tengan un mínimo impacto sobre el ambiente [46]. 


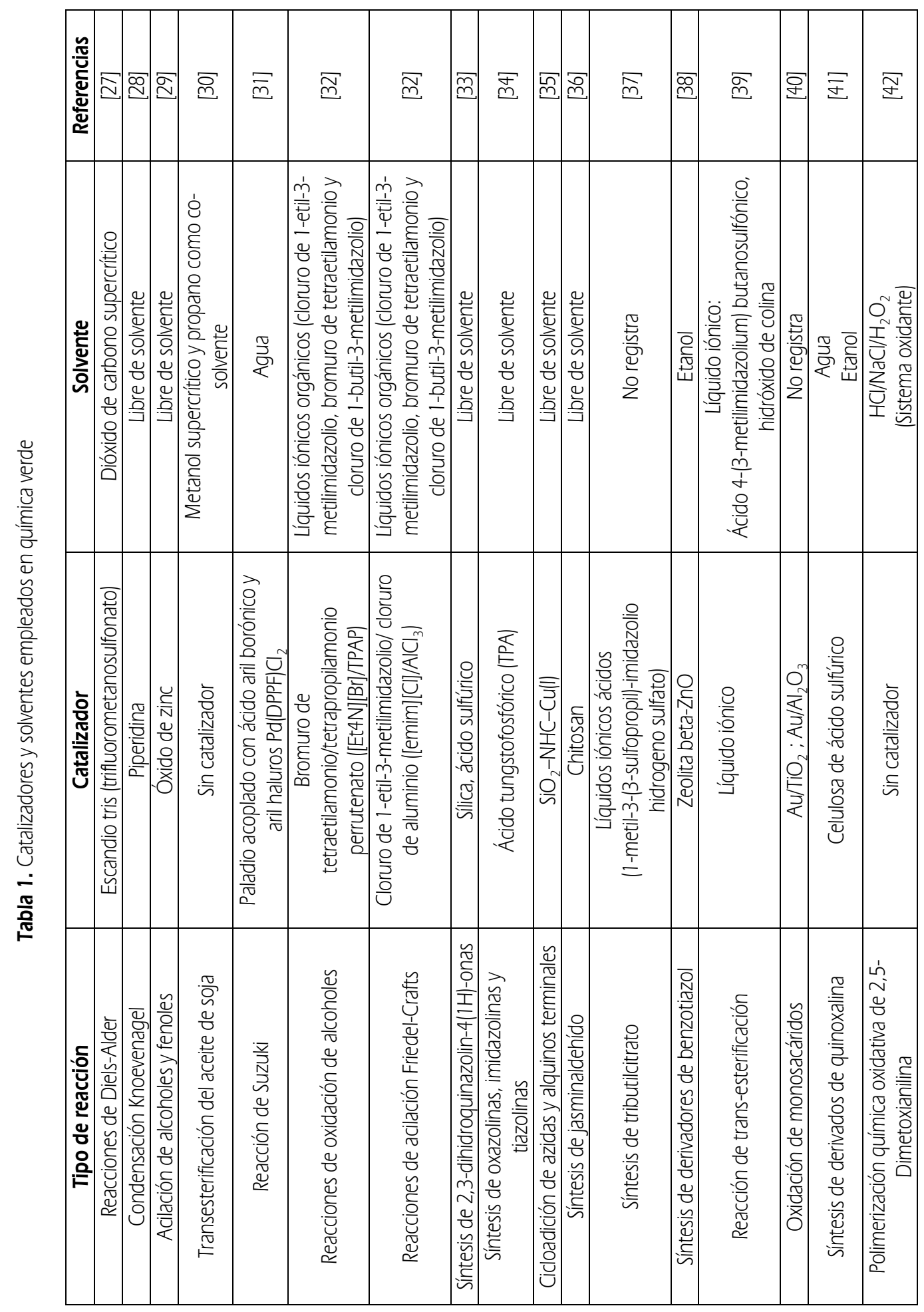


Por otra parte, precedentes en la industria química demuestran cómo el cambio estructural de una molécula puede mejorar su comportamiento ambiental sin reducir su funcionabilidad, como en el caso del tetrapropilenbencensulfonato (TPS), un surfactante que se usó en detergentes durante los años 50. Su biodegradablidad era baja y dio lugar a grandes formaciones de espuma en los rios y plantas de tratamiento de aguas residuales. Los químicos lograron solucionar este problema, transformando el TPS en sulfonato de alquil benceno lineal (LAS), que se degrada con mayor fácilidad [47].

El desarrollo de nuevos medicamentos y la síntesis de moléculas bioactivas implican procesos extremadamente costosos que incluyen la formulación, presentación final del fármaco y control de calidad en cada etapa de la manufactura del producto. Por lo tanto, la industria farmacéutica requiere rutas de síntesis amigables con el ambiente, sin dejar de cumplir con las demandas económicas y especificación de los productos. Además, se debe reducir continuamente los tiempos de producción de fármacos [48].

Las rutas de síntesis deben garantizar el cuidado del medio ambiente [48]. De hecho, un gran número de compañías farmacéuticas están reduciendo drásticamente la cantidad de residuos peligrosos en la manufactura de los productos farmacéuticos más vendidos. Pfizer, por ejemplo: rediseñó la síntesis de varios principios activos por medio de química verde, como el sildenafilo citrato, ingrediente activo de Viagra (R), y resultó una ruta de síntesis más eficiente que requiere menos trabajo y proporciona una mayor recuperación de solventes [4]. Del mismo modo, el proceso de fabricación del clorhidrato de sertralina, principio activo de Zoloft (R), fue rediseñado, siguiendo los principios de química verde [7] y [49]. GlaxoSmithKline, también modificó la ruta de síntesis del ácido 7-aminocefalosporánico (7-ACA), mediante la síntesis enzimática, reduciendo el número de etapas requeridas por la ruta de síntesis química [14] y [48].

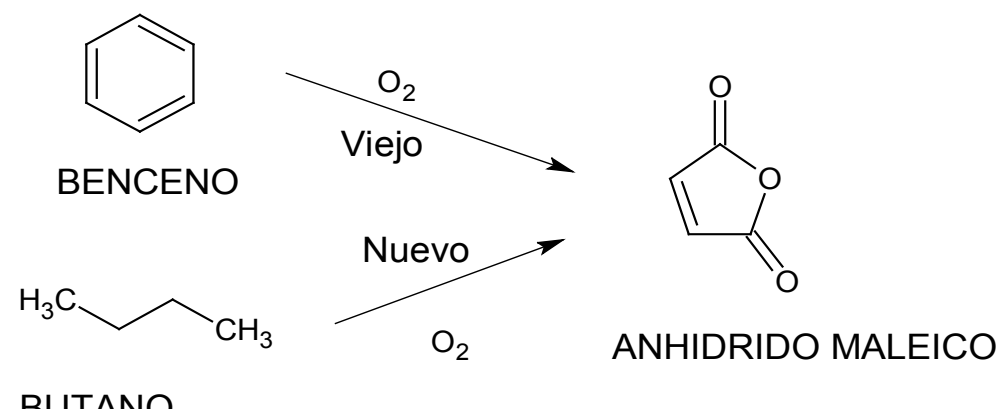

\section{BUTANO}

Figura 1. Ruta de síntesis antigua y nueva del anhídrido maleico.

Fuente: Centi G. et al [3]

En la actualidad, se han publicado innumerables estudios que proponen el uso de los principios de química verde. Un ejemplo es la síntesis del anhídrido maleico; el punto de partida de este proceso catalítico era el benceno, que fue reemplazado por butano, aplicando principios de la química verde, tales como el uso de reactivos no tóxicos, economía atómica mejorada, 
obtención del producto en una etapa sin uso de solventes y reducción en la producción de residuos (Figura 1) [3].

Otro ejemplo es la síntesis de ibuprofeno. La ruta sintética original involucra seis etapas con una eficiencia del 40\% y 60\% de residuos. En 1990, la compañía BHC rediseñó esta ruta y adoptó una estrategia muy diferente a la original con sólo dos etapas. La eficiencia fue incrementada en un $77 \%$ con la posibilidad de llegar a un 99\%, reciclando los subproductos de la primera reacción (el ácido acético puede ser fácilmente convertido en anhídrido acético, reactivo de la primera reacción). La ruta verde produce más ibuprofeno en menos tiempo y facilita la reducción de los requerimientos energéticos, en comparación con el proceso original (Figura 2) [1 1], [12] y [13].

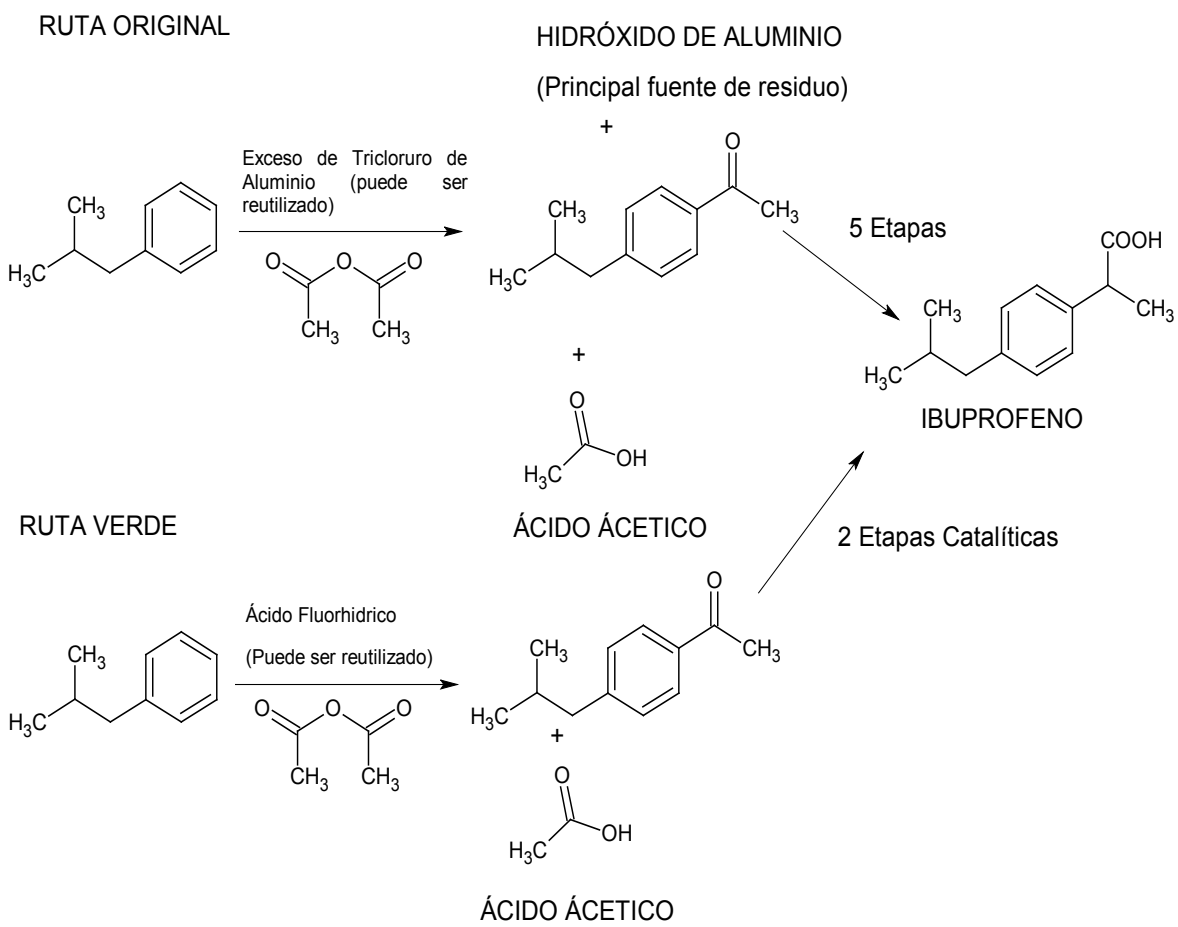

Figura 2. Ruta de síntesis del ibuprofeno.

Fuente: Anastas P. et al [1 1]

Debido a la regioselectividad y esteroselectividad de las enzimas, es posible realizar síntesis en donde se necesitan múltiples pasos de protección y desprotección, evitando reacciones o productos indeseados. Todas estas ventajas la convierten en una poderosa herramienta verde para la catálisis de procesos químicos [23]. Desde la década pasada, la industria ha estado orientándose hacia la aplicación de los principios de la química verde, introduciendo novedosos productos y tecnologías, usando solventes verdes y haciendo énfasis en la química enzimática o biocatálisis [1 1] y [43]. 
En virtud de que las funciones del cuerpo humano usan catálisis quiral, la tendencia dentro de la industria farmacéutica es buscar nuevos reactivos asimétricos [50]. En el momento, la síntesis de muchos intermediarios se realiza por biocatálisis, usando enzimas y microorganismos en fármacos para la ansiedad, hipertensión e insuficiencia cardíaca congestiva, antidiabéticos, antivirales, anticancerígenos, en drogas anticolesterol, drogas anti-Alzheimer y antibióticos, entre otros [51] y [52]. Un 35\% de los intermediarios de reacción en la industria farmacéutica, fueron quirales en el año 2000 [49], y se espera que aumente su uso debido a las ventajas que presenta. Las enzimas pueden usarse en la creación y resolución de centros quirales mediante reacciones asimétricas como la reducción con deshidrogenasas y la formación de enlaces C-C con liasas [48].

Por otra parte, laboratorios Lilly ganó un Premio de Investigación en química verde en 1999 [12], por rediseñar la síntesis de un fármaco con propiedades anticonvulsivantes, LY300164. Este agente farmacéutico está siendo desarrollado para el tratamiento de la epilepsia y enfermedades neurodegenerativas. La nueva síntesis utiliza la levadura zygosaccharomyces rouxii en un sistema de reacción que consta de tres fases, las cuales permiten la eliminación de los componentes de la reacción orgánica en los residuos acuosos. Un paso clave en la síntesis, fue una oxidación selectiva con aire comprimido que eliminó el uso de óxido de cromo, un posible carcinógeno. Sólo fue necesario aislar tres de los seis intermedios de síntesis, y la exposición de los trabajadores a agentes nocivos, y los costes del proceso fueron significativamente inferiores. El nuevo esquema sintético demostró ser más eficiente que el anterior, pasando de un rendimiento del 16\% al 55\% [12].

Los trabajos anteriormente mencionados, reflejan el gran impacto que tiene la aplicación de la química verde en la resolución de problemas ambientales, impulsando la práctica de una química que permite encontrar rutas y realizar procesos que disminuyan el impacto negativo que hasta ahora, se ha ejercido sobre el ambiente, premisa fundamental del desarrollo sostenible [53].

\section{RETOS PRESENTES Y FUTUROS}

El reto principal de la química verde es eliminar gradualmente la generación de materiales peligrosos o nocivos, y sustituirlos por otros menos tóxicos y más seguros. Sin embargo, este proceso debe ser impulsado con desarrollos científicos o tecnológicos y planteamientos de carácter legislativo. Su aplicación en el sector industrial, gubernamental y académico, ha generado múltiples beneficios ambientales, económicos y sociales. Aunque muchos procesos están todavía en fase de investigación, la magnitud del cambio es potencialmente promisoria y se observan resultados prometedores. Lo anterior implica un compromiso de todos los entes involucrados en este proceso, ya sea como ciudadanos, empresarios o como políticos, por lo cual la sociedad debe dar a conocer y comprender la aplicación de medidas a favor de la 
sostenibilidad que aunque conlleva a un coste económico a corto plazo, su beneficio a medio y largo plazo es muy promisorio.

Del diseño y la manipulación adecuada de productos químicos, así como de la reducción de las emisiones generadas por las industrias química y farmacéutica, depende en gran parte, el futuro del Planeta. Teniendo en cuenta que el desafío de la química verde es tan diverso como la imaginación científica, no es sorprendente que su aplicación involucre todos los sectores de la sociedad, desde la investigación hasta el gobierno y la educación. Esta última implica formar futuros ciudadanos con actitudes y comportamientos responsables en términos ambientales.

El crecimiento de la química verde en el transcurso de las últimas décadas ha aumentado a un ritmo acelerado, y sus avances continuarán hasta que sus 12 principios sean incorporados como parte de la química cotidiana. Todo lo cual constituye una plataforma necesaria para alcanzar el desarrollo sostenible.

\section{REFERENCIAS BIBLIOGRÁFICAS}

[1] Heine L. (2007). Sustainable materials and green chemistry, Access Science McGraw-Hill. En: http://mww.accessscience.com, Consultada el 27 de Julio del 2010.

[2] Rodríguez M., y Espinoza G. (2002). Gestión ambiental en América Latina y el Caribe; Capítulo 3: Problemas ambientales de la región. Banco Iberoamericano de Desarrollo. En: http://mww.iadb.or/sds/env, Consultada el 10 de Diciembre 2010.

[3] Centi G. and Perathonerm S. (2003). Catalysis and sustainable (green) chemistry. En: Catal. Today, vol.77, pp. 287-297.

[4] Kirchhoff M. (2005). Promoting sustainability through green chemistry. En: Resour. Conserv. Recy., Vol.44, pp. 237-243.

[5] García L. (2009). Biotecnología para una química verde, respetuosa con el medio ambiente, En: Revista de Prensa Tribuna Libre, En: http://mww.almendron.com/tribuna/24515/biotecnologia-para-una-quimica-verde/, Consultada el 10 Febrero del 2010.

[6] Anastas P., and Kirchhoff M. (2002). Origins, Current Status, and Future Challenges of Green Chemistry. En: Acc. Chem. Res., Vol.35, pp. 686-694.

[7] Manley J., Anastas P., and Cue B. (2008). Frontiers in Green Chemistry: meeting the grand challenges for sustainability in R\&D and manufacturing. En: J. Cleaner Prod., Vol.16, pp. 743-750. 
[8] García J., Pérez L., and Cocero M. (2007). New trends for design towards sustainability in chemical engineering: Green engineering. En: Chem. Eng. J., Vol.133, pp. 7-30.

[9] Vargas E., y Ruiz L. (2007). Química Verde en el siglo XXI: Química Verde, Una Química Limpia. En: Revista Cubana de Química, Vol. XIV, pp. 29-32.

[10] Vilches A., y Pérez D. (2011). Papel de la Química y su enseñanza en la construcción de un futuro sostenible. En: Educ. quím. Versión digital, pp. 1-14.

[11] Anastas P., Kirchhoff M., and Williamson T. (2001). Catalysis as a foundational pillar of green chemistry. En: Appl. Catal., A, Vol.221, pp. 3-13.

[12] Warner J., Cannon A., and Dye K. (2004). Green chemistry. En: Environ. Impact Assess. Rev., Vol.24, pp. 775-799.

[13] Poliakoff M., and Licence P. (2007). Sustainable Technology: Green chemistry. En: Nature, Vol.450, pp. 1.

[14] Cabri W. (2009). The pharmaceutical perspective. En: Catal. Today, Vol.140, pp. 2-10.

[15] Hutchings G. (2007). A golden future for green chemistry. En: Catal. Today, Vol. 122, pp. 196-200.

[16] Anastas P., Bartlett L., Kirchhoff M., and Williamson T. (2000) The role of catalysis in the design, development, and implementation of green chemistry. En: Catal. Today, Vol.55, pp. 11-22.

[17] Kümmerer K. 2007). Sustainable from the very beginning: rational design of molecules by life cycle engineering as an important approach for green pharmacy and green chemistry. En: Green Chem., Vol.9, pp. 899-907.

[18] Talawar M.B., Sivabalan R., Mukundan T., Muthurajan H., Sikder A.K., Gandhe B.R., and Subhananda R.A.J. (2009). Environmentally compatible next generation green energetic materials (GEMs). En: Hazard. Mater. Vol. 161, pp. 589-607.

[19] Clark J. (2001). Catalysis for green chemistry. En: Pure Appl. Chem., Vol.73, pp. 103-111.

[20] Li C., and Trost B. (2008) Green chemistry for chemical synthesis. En: Proc. Natl. Acad. Sci. U S A., Vol.105, pp. 13197-13202.

[21] Clark J. (1999). Green chemistry: challenges and opportunities, Green Chem. En: www.rsc.org/delivery/_ArticleLinking/DisplayArticleForFree.cfm?doi=a807961 g, Página consultada el 20 Enero del 2011. 
[22] Misono M. (2000). Acid catalysts for clean production Green aspects of heteropolyacid catalysts. En: C. R. Acad. Sci. Paris, Serie Ilc, Chimie. Vol.3, pp. 471-475.

[23] Lozano P. (2010). Enzymes in neoteric solvents: From one-phase to multiphase systems. En: Green Chem., Vol.12, pp. 555-569.

[24] Noyori R. (2010). Insight: Green chemistry: the key to our future En: Tetrahedron, Vol.66, $1028 \mathrm{p}$.

[25] Folié M., Gani R., Jímenez C., and Constable D. (2008). Systematic Selection of Green Solvents for Organic Reacting Systems. En: Chin. J. Chem. Eng., Vol. 16, pp. 376-383.

[26] Centi G., Ciambelli P., Perathoner S., and Russo P. (2002). Environmental catalysis: trends and Outlook. En: Catal. Today, Vol.75, pp. 3-15.

[27] Leitner W. (2000). Homogeneous catalysts for application in supercritical carbon dioxide as a 'green' solvent. En: C. R. Acad. Sci. Paris, Serie Ilc, Chimie., Vol.3, pp. 595-600.

[28] McCluskey A., Robinson P., Hill T., Scott J., and Edwards K. (2002). Green chemistry approaches to the Knoevenagel condensation: comparison of ethanol, water and solvent free (dry grind) approaches. En: Tetrahedron Lett., Vol.43, pp. 3117-3120.

[29] Tamaddon F., Amrollahi M., and Sharafat L. (2005) A green protocol for chemoselective O-acylation in the presence of zinc oxide as a heterogeneous, reusable and eco-friendly catalyst. En: Tetrahedron Lett. Vol. 46, pp.7841-7844.

[30] Cao W., Han H., and Zhang J. (2005) Preparation of biodiesel from soybean oil using supercritical methanol and co-solvent. En: Fuel, Vol. 84, pp. 347-351.

[31] Jiang N., and Ragauskas A. (2006) Environmentally friendly synthesis of biaryls: Suzuki reaction of aryl bromides in water at low catalyst loadings. En: Tetrahedron Lett. Vol.47, pp. 197-200.

[32] Boschetti A., Montagnaro F., Rienzo C., and Santoro L. (2007) A preliminary investigation on the use of organic ionic liquids as green solvents for acylation and oxidation reactions. En: J. Cleaner Prod., Vol.15, pp. 1797-1805.

[33] Dabiri M., Salehi P., Baghbanzadeh M., Zolfigol M., Agheb M., and Heydari S. (2008). Silica sulfuric acid: An efficient reusable heterogeneous catalyst for the synthesis of 2,3dihydroquinazolin-4(1H)-ones in water and under solvent-free conditions. En: Catal. Commun. Vol.9, pp. 785-788. 
[34] Mohammadpoor-Baltork I., Moghadam M., Tangestaninejad S., Mirkhani V., and Fatemeh S. (2008). Environmental-friendly synthesis of oxazolines, imidazolines and thiazolines catalyzed by tungstophosphoric acid. En: Catal. Commun. Vol.9, pp. 11531161.

[35] Pinhua L., Lei W., and Yicheng Z. (2008). $\mathrm{SiO}_{2}-\mathrm{NHC}-\mathrm{Cu}(\mathrm{l})$ : an efficient and reusable catalyst for [3+2] cycloaddition of organic azides and terminal alkynes under solvent-free reaction conditions at room temperature. En: Tetrahedron, Vol.64, pp. 10825-10830.

[36] Sudheesh N., Sharma S., and Shukla R. (2008). Chitosan as an eco friendly solid base catalyst for the solvent-free synthesis of jasminaldehyde. En: J. Mol. Catal. A: Chem., Vol.312, pp. 77-82.

[37] Junming X., Jianchun J., Zhiyue Z., and Jing L. (2010). Synthesis of tributyl citrate using acid ionic liquid as catalyst. En: Process Saf. Environ. Prot., Vol.88, pp. 28-30.

[38] Katkar S., Mohite P., Gadekar L., Vidhate K., and Lande M. (2010). ZnO-beta zeolite: As an effective and eco-friendly heterogeneous catalyst for the synthesis of benzothiazole derivatives. En: Chin. Chem. Lett., Vol.21, pp. 421-425.

[39] Earle M.J., Plechkova N.V., and Seddon K.R. (2009). Green synthesis of biodiesel using ionic liquids. En: Pure Appl. Chem., Vol.81, pp. 2045-2057.

[40] Mirescu A., and Prüße U. (2007). New environmental friendly method for the preparation of sugar acids via catalytic oxidation on gold catalysts. En: Appl. Catal., B, Vol.70, pp. 644652.

[41] Shaabani A., Rezayan A.H., Behnam M., and Heidary M. (2009). Green chemistry approaches for the synthesis of quinoxaline derivatives: Comparison of ethanol and water in the presence of the reusable catalyst cellulose sulfuric acid. En: C. R. Chim., Vol. 12, pp. $1249-1252$.

[42] Jain S., Surwade P.S., Agnihotra S.R., Dua V., Eliason P.A., Morose G.J. and Manohar S.K. (2010) Green chemistry synthesis of nanostructured poly (2,5-dimethoxyaniline). En: Green Chem., Vol.12, pp. 585-589.

[43] Kang E. (2010). Commentary: an industrial perspective on green chemistry. En: Tetrahedron, Vol.66, pp. 1029-1030.

[44] Takagaki A., Tagusagawa C., Hayashi S., Hara M., and Domen K. (2010) Nanosheets as highly active solid acid catalysts for green chemical syntheses. En: Energy Environ. Sci., Vol.3, pp. 82-93. 
[45] Mallakpour S., and Taghavi M. (2009). Direct polyamidation in green media: Studies on thermal degradation of novel organosoluble and optically active flame retardant polyamides. En: React. Funct. Polym. Vol.69, pp. 206-215.

[46] Hardy J., Green chemistry chapter. En: http://mww.rsc.org/images/Chap8_tcm18108598.pdf, Consultada el 14 de Enero del 2011.

[47] Everts S. (2010) Greener Drugs: Benign-By-Design Strategies. En: Chemical and Engenieering News., vol.88. En: http://pubs.acs.org/cen/science/88/8813scila.html, Consultada el 11 de Junio del 2010

[48] Woodley J. (2008). New opportunities for biocatalysis: making pharmaceutical processes greener. En: Trends Biotechnol. Vol.26, pp. 321-327.

[49] Nameroff T., Garant R., Albert M. (2004). Adoption of green chemistry: an analysis based on US patents. En: Resour. Policy. Vol.33, pp. 959-974.

[50] Pollard D., Woodley J. (2007). Biocatalysis for pharmaceutical intermediates: the future is now. En: Trends Biotechnol. Vol.25, pp. 66-73.

[51] Ramesh P.N. (2008). Synthesis of chiral pharmaceutical intermediates by biocatalysis. En: Coord. Chem. Rev. Vol.252, pp. 659-701.

[52] Ahmad A.L., On P.C., Abd Shukor S.R. (2009). Sustainable biocatalytic synthesis of Lhomophenylalanine as pharmaceutical drug precursor. En: Biotechnol. Adv. Vol.27, pp. 286-296.

[53] Morales M., Martínez J., Reyes-Sánchez L., Martín O., Arroyo G., Obaya A., Miranda R. (2011). ¿Qué tan verde es un experimento?. En: Educ. quím., Vol. 22, pp. 240-248. 